\title{
QUEEN'S
UNIVERSITY
BELFAST
}

\section{The Sporadic Activity of (6478) Gault: A YORP-driven event?}

Kleyna, J. T., Hainaut, O. R., Meech, K. J., Hsieh, H. H., Fitzsimmons, A., Micheli, M., Keane, J. V., Denneau, L., Tonry, J., Heinze, A., Bhatt, B. C., Sahu, D. K., Koschny, D., Smith, K. W., Ebeling, H., Weryk, R., Flewelling, H., \& Wainscoat, R. J. (2019). The Sporadic Activity of (6478) Gault: A YORP-driven event? Astrophysical Journal Letters, 874(2), [L20]. https://doi.org/10.3847/2041-8213/ab0f40

Published in:

Astrophysical Journal Letters

Document Version:

Publisher's PDF, also known as Version of record

Queen's University Belfast - Research Portal:

Link to publication record in Queen's University Belfast Research Portal

Publisher rights

(c) 2019. The American Astronomical Society. All rights reserved. This work is made available online in accordance with the publisher's policies. Please refer to any applicable terms of use of the publisher.

\section{General rights}

Copyright for the publications made accessible via the Queen's University Belfast Research Portal is retained by the author(s) and / or other copyright owners and it is a condition of accessing these publications that users recognise and abide by the legal requirements associated with these rights.

Take down policy

The Research Portal is Queen's institutional repository that provides access to Queen's research output. Every effort has been made to ensure that content in the Research Portal does not infringe any person's rights, or applicable UK laws. If you discover content in the Research Portal that you believe breaches copyright or violates any law, please contact openaccess@qub.ac.uk. 


\title{
The Sporadic Activity of (6478) Gault: A YORP-driven Event?
}

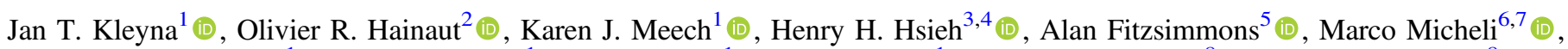

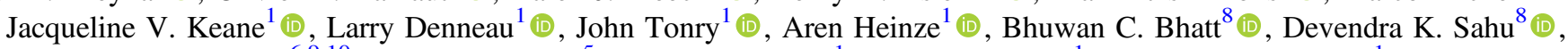

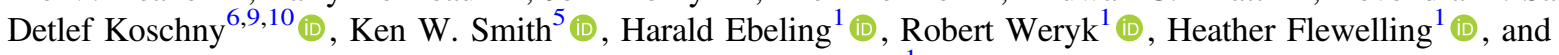 \\ Richard J. Wainscoat ${ }^{1}$ (1) \\ ${ }^{1}$ Institute for Astronomy, University of Hawaii, 2680 Woodlawn Drive, Honolulu, HI 96822, USA \\ ${ }^{2}$ European Southern Observatory, Karl-Schwarzschild-Strasse 2, D-85748 Garching bei München, Germany \\ ${ }^{3}$ Planetary Science Institute, 1700 East Fort Lowell Road, Suite 106, Tucson, AZ 85719, USA \\ ${ }^{4}$ Institute of Astronomy and Astrophysics, Academia Sinica, P.O. Box 23-141, Taipei 10617, Taiwan \\ ${ }^{5}$ Astrophysics Research Centre, Queen's University Belfast, Belfast BT7 1NN, UK \\ ${ }^{6}$ ESA NEO Coordination Centre, Largo Galileo Galilei, 1, I-00044 Frascati (RM), Italy \\ ${ }^{7}$ INAF-Osservatorio Astronomico di Roma, Via Frascati, 33, I-00040 Monte Porzio Catone (RM), Italy \\ ${ }^{8}$ Indian Institute for Astrophysics, II Block, Koramangala, Bangalore 560 034, India \\ ${ }^{9}$ ESTEC, European Space Agency, Keplerlaan 1, 2201 AZ, Noordwijk, The Netherlands \\ ${ }^{10}$ Chair of Astronautics, Technical University of Munich, Boltzmannstraße 15, D-85748 Garching bei München, Germany \\ Received 2019 February 22; revised 2019 March 13; accepted 2019 March 13; published 2019 April 1
}

\begin{abstract}
On 2019 January 5 a streamer associated with the $4-10 \mathrm{~km}$ main belt asteroid (6478) Gault was detected by the ATLAS sky survey, a rare discovery of activity around a main belt asteroid. Archival data from ATLAS and PanSTARRS1 show the trail in early 2018 December, but not between 2010 and 2018 January. The feature has significantly changed over one month, perfectly matching predictions of pure dust dynamical evolution and changes in the observing geometry for a short release of dust around 2018 October 28. Follow-up observations with the Hubble Space Telescope (HST) show a second narrow trail corresponding to a brief release of dust on 2018 December 30. Both releases occurred with negligible velocity. We find the dust grains to be fairly large, with power-law size distributions in the $10^{-5}-10^{-3} \mathrm{~m}$ range and power-law indices of $\sim-1.5$. Three runs of groundbased data find a signature of $\sim 2 \mathrm{hr}$ rotation, close to the rotational limit, suggesting that the activity is the result of landslides or reconfigurations after Yarkovsky-O'Keefe-Radzievskii-Paddack (YORP) spin-up.
\end{abstract}

Key words: minor planets, asteroids: individual ((6478) Gault) - planets and satellites: dynamical evolution and stability

\section{Introduction-Active Asteroids}

Active asteroids are objects that have semimajor axes smaller than Jupiter's, are orbitally decoupled from Jupiter (with Tisserand parameter $T_{J}>3.0$ ), and exhibit comet-like mass loss (Jewitt et al. 2015). They are dynamically distinct from classical comets and have long been present in the outer asteroid belt (Kresak 1980; Levison et al. 2006). Many mechanisms have been proposed to explain the dust observed around active asteroids, including rotational spin-up (Yarkovsky-O'Keefe-Radzievskii-Paddack (YORP); e.g., Bottke et al. 2006; Vokrouhlický et al. 2015), asteroid impact, collisional debris fields, and sublimation of subsurface ices in main belt comets (MBCs; Jewitt et al. 2015). Active asteroids offer insight into a range of solar system phenomena (primordial volatiles from MBC sublimation, material composition from rotation and impacts), and it is crucial to study each specimen in detail to determine its mechanism of activity.

\section{A New Active Asteroid: (6478) Gault}

The Hawai'i ATLAS survey (Tonry et al. 2018) detected a tail (Figure 1) on asteroid (6478) Gault in images obtained on 2019 January 5, when the object was at a heliocentric distance of $r=2.48$ au (Smith \& Denneau 2019). A median-combined stack of seven $30 \mathrm{~s}$ exposures shows a 135"-long tail at $\mathrm{PA}=290^{\circ}$. The ATLAS archive shows that Gault was active on 2018 December 8, with a $30^{\prime \prime}$ tail at PA $=290^{\circ}$. However, we find no evidence of a tail in previous ATLAS images obtained during 2018 January (it was not observed by ATLAS or Pan-STARRS from 2018 January through December because of its low solar elongation). Ye et al. (2019) find evidence of the onset, reporting Zwicky Transient Facility (ZTF) archive data showing significant brightening before 2018 October 31.

The orbital elements $(e=0.194, a=2.305$ au, $i=22.8$, having $T_{J}=3.461$ ) are consistent with it being an MBC, albeit with a small semimajor axis. Discovered in 1988, Gault has an absolute magnitude of 14.4 in the $V$ band, based on $\sim 1000$ survey observations, implying a diameter of $\sim 9 \mathrm{~km}$, assuming a $4 \%$ geometric albedo typical of comets, or $4 \mathrm{~km}$ for a $20 \%$ albedo, representative of asteroids; we will assume a $20 \%$ albedo unless otherwise stated. Gault's tail was also seen in Pan-STARRS1 survey images from 2018 December 17 but appeared stellar in all other images from 2010 September 6 through 2018 January 11, implying that something in late 2018 triggered the activity. A second emission event detected in midJanuary in our data, and reported by Ye et al. (2019) and Jehin et al. (2019), shows similarities to the episodic activity of 311P, attributed to landslides caused by rotational instability (Jewitt et al. 2018).

\section{Follow-up Observations}

Images from the Canada-France-Hawaii Telescope (CFHT) $3.6 \mathrm{~m}$ telescope on 2019 January 6 show a tail $\gtrsim 4.3 \times 10^{5} \mathrm{~km}$ long. The central brightness measured with both ATLAS and CFHT is more than a magnitude brighter than predicted by Jet 


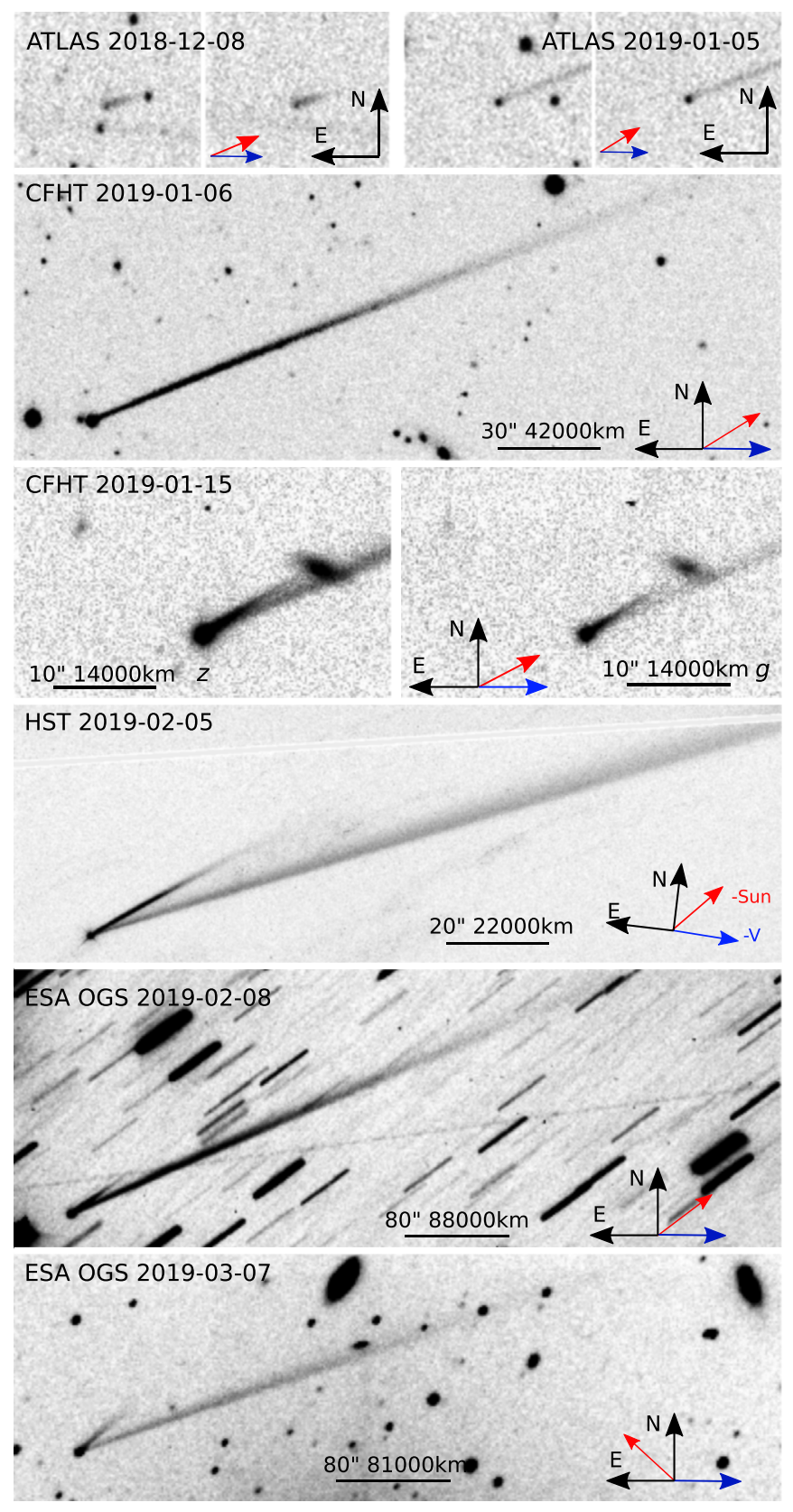

Figure 1. Evolution of the (6478) Gault dust tail from the initial discovery in the ATLAS data through the first HST visit on 2019 February 5. The tail on 2019 January 6 obtained with the CFHT $3.6 \mathrm{~m}$ telescope was $310^{\prime \prime}\left(4.3 \times 10^{5}\right.$ $\mathrm{km})$ long. The arrows indicate the orientation of the field and the antisolar direction and the negative of the object's velocity. The images are individually adjusted on a negative logarithmic scale.

Propulsion Laboratory (JPL) Horizons, implying significant excess material within the ground-based seeing disk. Other groups also reported an extended tail (Maury et al. 2019). Both a set of 17120 s Sloan Digital Sky Survey (SDSS) g, r, i, z CFHT images on 2019 January 15, and a series of 58 SDSS- $r^{\prime}$ band $120 \mathrm{~s}$ images on 2019 January 24 with the $2.54 \mathrm{~m}$ Isaac Newton Telescope (INT) on La Palma, Spain, clearly showed both the previously reported tail and a new short dust tail subsequently reported by Jehin et al. (2019).

We were allocated three one-orbit observations with the Hubble Space Telescope (HST; program GO/DD-15678) to study the evolution and morphology of the dust trail at high resolution and to search for possible fragments, with the goal of identifying the cause of the mass loss from the competing scenarios of sublimation, impact disruption, or YORP spin-up. The first visit for this program was executed on 2019 February 5 , yielding five dithered images of $380 \mathrm{~s}$ duration through the F350LP filter (WFC3/UVIS); Figure 1 shows the stacked composite.

To assess Gault's rotation period, photometric data were obtained with the $1 \mathrm{~m}$ European Space Agency Optical Ground Station (OGS) at the Teide Observatory, Tenerife, on 2019 February 8. One hundred fifty exposures of $90 \mathrm{~s}$ were obtained between 00:30 and 04:30 UT with a signal-to-noise ratio $(\mathrm{S} / \mathrm{N}) \gtrsim 100$.

Additional data were obtained on 2019 February 10 with the $2 \mathrm{~m}$ Himalayan Chandra Telescope (HCT) located at HanleLadakh, yielding $76 R$-band images of $120 \mathrm{~s}$, with mostly modest extinction of $\lesssim 0.5 \mathrm{mag}$, but occasional highs of $\sim 2$ mag.

Finally, a 351 exposure, $5.6 \mathrm{hr}$ sequence of SDSS- $r^{\prime}$ band images was obtained on 2019 February 18 with the $4.2 \mathrm{~m}$ William Herschel Telescope (WHT) on La Palma. Conditions were photometric, but the images suffered from seeing of $\sim 1 "$.5-2." 0 , and from non-uniform scattered light from the Moon, $29^{\circ}$ away. Table 1 lists the follow-up observations.

\section{Dust Dynamical Model}

A Finson-Probstein (FP; Finson \& Probstein 1968; Farnham 1996) dust analysis calculates the trajectories of dust grains of different sizes, parameterized by $\beta$ (the ratio of radiation force and solar gravity) ejected from the asteroid's surface at different times, $t$, as acted upon by solar gravity and solar radiation pressure. We used the FP approach to compare synchrones (loci of the particles emitted at the same time $t$ ) and syndynes (curves joining particles with the same $\beta$ ) to HST images of Gault's dust environment (see Figure 2). $\beta$ is related to the size of dust grains by

$$
\beta=5.740 \times 10^{-4} \times \frac{Q_{\mathrm{pr}}}{\rho a},
$$

where $Q_{\mathrm{pr}}$ is a radiation-pressure efficiency coefficient $(\sim 1-2$ for rocky and icy material), $\rho$ is the density, and $a$ is the grain size. For $\rho=3000 \mathrm{~kg} \mathrm{~m}^{-3}$ and $Q_{\mathrm{pr}}=1.05$, Equation (1) yields $a=2 \times 10^{-7} \beta^{-1}[\mathrm{~m}]$.

The dust emission started abruptly at $t-102$ days (2018 October 26) before the HST observations $(t=0)$, in agreement with the ZTF observations of Ye et al. (2019). No dust is observed on older synchrones, and the boundary of the trail matches the synchrone perfectly, indicating a sharply defined event (i.e., shorter than the resolution of the FP modeling, 1 day) and a broad distribution of large dust grains, with $\beta<0.01$ or $a>20 \mu \mathrm{m}$. Smaller dust grains were pushed out of the field of view. The dust emission peaks at $t-100$ days (2018 October 28), then decreases to almost no dust at $t-85$ days (2018 November 12). However, while the onset of the activity is sharply delimited by the $t=102$ day synchrone, the turnoff is not precisely aligned with the $t-85$ day synchrone. The streamer has a fairly constant width, while the distance between the synchrones increases radially, indicative of a small ejection velocity.

The data show that a second episode of dust emission took place around day $t-37$ (2018 December 30, \pm 1 day). This 
Table 1

Observations

\begin{tabular}{|c|c|c|c|c|c|c|c|c|c|c|}
\hline Telescope & UT Date & $\operatorname{Exp}^{a}$ & \#Еxp & Filter & Sky & Seeing & $r^{\mathrm{b}}$ & $\Delta^{\mathrm{b}}$ & $\alpha^{\mathrm{b}}$ & $\mathrm{TA}^{\mathrm{b}}$ \\
\hline CFHT MCam & 2019 Jan 15 & 120 & 17 & $\mathrm{~g}, \mathrm{r}, \mathrm{i}, \mathrm{z}$ & clear & 0.6 & 2.47 & 1.88 & 21.0 & 238.0 \\
\hline CFHT MCam & 2019 Feb 6 & $60-120$ & 42 & $\mathrm{~g}, \mathrm{r}, \mathrm{i}, \mathrm{z}, \mathrm{w}$ & clear & 1.2 & 2.46 & 1.77 & 19.4 & 240.2 \\
\hline INT & 2019 Jan 23 & 120 & 58 & $\mathrm{r}$ & clear & 2.0 & 2.44 & 1.68 & 17.6 & 242.0 \\
\hline ESA OGS & 2019 Feb 8 & 90 & 150 & Open & clear & 2.0 & 2.41 & 1.52 & 12.5 & 246.1 \\
\hline HCT 2 m & 2019 Feb 10 & 120 & 76 & $\mathrm{R}$ & cloud & 2.0 & 2.40 & 1.50 & 11.8 & 246.6 \\
\hline WHT & 2019 Feb 19 & 40 & 350 & $\mathrm{r}$ & clear, moon & 1.7 & 2.38 & 1.44 & 8.7 & 248.9 \\
\hline
\end{tabular}

Notes.

a Image exposure time (s).

${ }^{\mathrm{b}}$ Heliocentric and geocentric distance (au), phase angle (deg), and true anomaly (deg)

episode was short and peaked, with a FWHM of 1 day; however, Ye et al. (2019) suggest that the event developed over several days. While this trail is much shorter than the first, it shows smaller dust ( $a \gtrsim 5 \mu \mathrm{m})$, as the grains have had less time to be removed by radiation pressure. No dust is visible on the synchrones ranging between the second peak and the time of the observation.

The dust synchrone orientations for the ATLAS observations were $\mathrm{PA}=288^{\circ}$ for 2018 December 8 and $\mathrm{PA}=291^{\circ}$ for 2019 January 5 (in excellent agreement with the ATLAS reported directions $\mathrm{PA}=290^{\circ}$ and $\mathrm{PA}=291^{\circ}$, respectively). The computed trail lengths out to $\beta=0.02$ were $32^{\prime \prime}$ and $120^{\prime \prime}$, versus $30^{\prime \prime}$ and $135^{\prime \prime}$ reported from the observations. Thus the dust trail reported from the ATLAS observations matches the trail corresponding to the first release of dust on 2018 October 28.

The second dust release on 2018 December 30 was present in the 2019 January 5 ATLAS and January 6 and 8 CFHT observations, but not identified as a trail. Its presence explains the reported $>1$ magnitude excess in the seeing disk.

The area around the nucleus is devoid of dust, indicating that the dust was released with tiny initial velocity. No fragments are visible down to $\sim 50 \mathrm{~m}$ radius.

Profiles were extracted along the synchrones over both trails. The value of $t$ is obtained from the PA of the synchrone. The linear position along these profiles were converted into $\beta$ using the FP model, and into grain radius using Equation (1). The flux of a grain of radius $a$ is estimated as

$$
f=10^{-0.4\left(M_{\odot}-\mathrm{ZP}\right)} p\left(\frac{a}{r \Delta}\right)^{2},
$$

where $f$ is the flux in CCD adu pixel ${ }^{-1} \mathrm{~s}^{-1}, M_{\odot}$ is the absolute magnitude of the Sun in the filter, $\mathrm{ZP}=26.817$ is the photometric zero-point, ${ }^{11} p=0.2$ is the dust albedo, $a$ is the radius, and $r$ and $\Delta$ are the helio- and geocentric distances (all in astronomical units).

These distributions show a dispersion, suggesting that the conversion from the position along the synchrone to $\beta$ and $a$ is not perfect. The same exercise was repeated, this time fixing the value of $t$ to the peaks of the streamers at 102 and 37 days. The resulting sharper distributions are shown in Figure 2(c). This suggests that the azimuthal spread of the streamer is dominated by an initial velocity rather than by a spread in emission time, independently supporting the earlier conclusion, based on the rectangular shape of the first streamer, that the

\footnotetext{
11 http://www.stsci.edu/hst/wfc3/analysis/uvis_zpts/uvis1_infinite
}

azimuthal spread was caused by a distribution of initial velocities rather than purely by dust dynamics. The direction of the emission velocity is not known, but the improvement of the profiles using a constant $\beta$ suggests that a velocity perpendicular to the streamer is a good approximation. This neglects a component perpendicular to the plane of the sky, which cannot be estimated. The spread of the streamer measured perpendicularly to its length $(l=5700 \mathrm{~km})$ and the age of the streamer $(t \sim 102$ days $)$ give a lower limit of $v_{e}=0.7 \mathrm{~m} \mathrm{~s}^{-1}$ for the maximum emission velocity.

A power law of $f(a) d a=C a^{n} d a$ was fitted to grain size distribution profiles, resulting in indices of $n=-1.70 \pm 0.08$ and $-1.64 \pm 0.01$ over the linear ranges for the first and second release events, respectively. The value of $a$ and the number of grains of that size are affected by the assumed values of the density $\rho$ and the albedo $p$, but the index of the power law is not.

In comparison, the streamers of activated asteroid $311 \mathrm{P} /$ 2013 P5 (Pan-STARRS) had a power-law index of -1.0 (Hainaut et al. 2014). Traditional sublimating comets have indexes in the -4 to 0.0 range (Sekanina 1980; Fulle et al. 2000; Moreno et al. 2016, 2017).

The number of grains in each pixel can also be used to estimate the mass of the dust in the streamers: with the same assumptions $\left(p=0.2, \quad \rho=3000 \mathrm{~kg} \mathrm{~m}^{-3}\right)$, this results in $m=7 \times 10^{9}$ and $4 \times 10^{7} \mathrm{~kg}$ for the first and second streamers, respectively, integrating over $a$ from $30 \mu \mathrm{m}$ to $2 \mathrm{~mm}$. These are lower limits, as the mass of the streamer is dominated by the large particles, for which the radiation pressure has not dispersed much and whose photometric contribution is small. The smaller particles, while more numerous, do not contribute much to the total mass (see Hainaut et al. 2012, Figure 10 for a quantitative discussion). To put these values in perspective, they correspond to spheres of 82 and $14 \mathrm{~m}$ radii, small compared to the bulk of the body. The mass in the two main trails of P/2013 P5, another active asteroid which presented similar morphology, was estimated using a similar method to $3 \times 10^{6} \mathrm{~kg}$ and $3 \times 10^{7} \mathrm{~kg}$ (Hainaut et al. 2014). P/2012 A2, also an active asteroid, presented a trail with a different morphology whose mass was estimated to be $8 \times 10^{8} \mathrm{~kg}$ (Hainaut et al. 2012).

\section{Rotation Period}

Using the OGS, HCT, and WHT data, we performed an analysis of Gault's light curve to determine the rotation period. The presence of a dust coma necessitated a small $2^{\prime \prime}$ aperture, which made our analysis susceptible to seeing variations. 

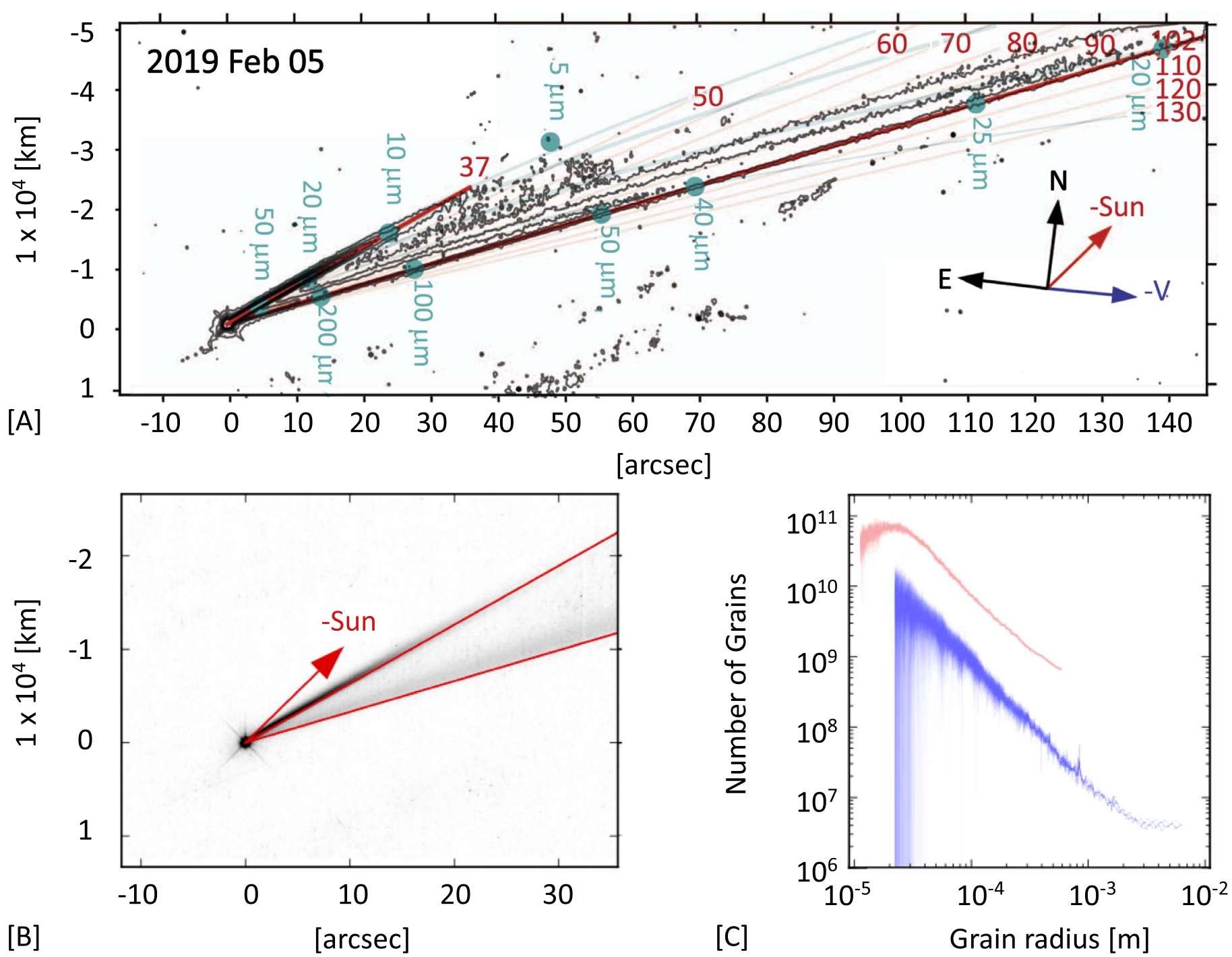

Figure 2. (A) Syndynes (blue) and synchrones (red, labeled in days before the observation date) for Gault $H S T$ data, $t=2019$ February 5 . The thicker synchrones mark the sharp onset of the dust emission ( $t-102$ days) and the peak of the secondary emission ( $t-37$ days). The corresponding grain radii are marked in blue. The orientation of the field and the antisolar and antivelocity vectors are shown. (B) Close-up view of (A). The two red lines are the synchrones corresponding to emissions at $t-102$ and $t-37$ days. The arrow marks the antisolar direction, the direction toward which dust would be drifting if emitted at the time of the observations. The lack of dust between the 37 day synchrone and the arrow indicates that the activity essentially stopped. No diffuse coma nor fragments are visible around the nucleus. (C) Distribution of the grain sizes around the 102 (blue) and 37 day (red) synchrones.

Nevertheless, after linear detrending all three data sets showed a $\sim 1 \mathrm{hr}$ signature in the spectral analysis, in agreement with a two-peaked $\sim 2 \mathrm{hr}$ rotation period. This is close to the critical breakup limit of a strengthless rubble pile $(\sim 3.3 \mathrm{hr}$ for a cometary object, and $1.9 \mathrm{hr}$ for an asteroid, with an absolute magnitude of $V=14.4$ ) and at the observed $2 \mathrm{hr}$ spin limit of asteroids (e.g., Pravec et al. 2002).

Figure 3 shows the data sets in the top three panels and the Lomb-Scargle spectral power (Lomb 1976) in the bottom panels, with Monte Carlo resamplings. The spectral peaks have formal significances of $p=1 \times 10^{-9}$ (WHT, $1.14 \mathrm{hr}$ period), $p=6 \times 10^{-3}(\mathrm{HCT}$, for the shorter $1.16 \mathrm{hr}$ period $)$, and $p=2 \times 10^{-5}$ (OGS, $0.97 \mathrm{hr}$ period). However, phasing and smoothing the data does not reveal any obvious light curve, suggesting that the periodic signal is buried in aperiodic, nonGaussian noise, and has low amplitude (perhaps $\lesssim 0.05 \mathrm{mag}$ ). A small amplitude light curve is expected if the dust coma contributes most of the flux (e.g., Hsieh et al. 2011), a supposition supported by the $\sim 1$ mag brightening noted above.

Repeating the test with a two-Fourier-component Analysis of Variance (ANOVA) using the PERANSO package (Paunzen \& Vanmunster 2016) also finds a $2 \mathrm{hr}$ period. A joint analysis of the temporally proximate WHT and ESA sets in PERANSO also shows a $2 \mathrm{hr}$ rotation, although the signal in the OGS set must be scaled up, as might be required of a signal masked by dust in the large 1". 4 pixels of OGS. The INT data, spanning $3 \mathrm{hr}$, also had a broad but insignificant $2 \mathrm{hr}$ rotation. Although the absence of a visible light curve precludes definitive conclusions about the period, the presence of a $2 \mathrm{hr}$ rotational signature in three distinct data sets, under two methods of analysis, with robustness under Monte Carlo resampling, is persuasive.

An analysis of the 323 sparse observations found in ATLAS from 2016 to 2018 did not detect a signal; however, ATLAS cannot rule out variations with an amplitude of $\leqslant 0.05 \mathrm{mag}$, in accord with the low amplitude inferred from our optical data. 

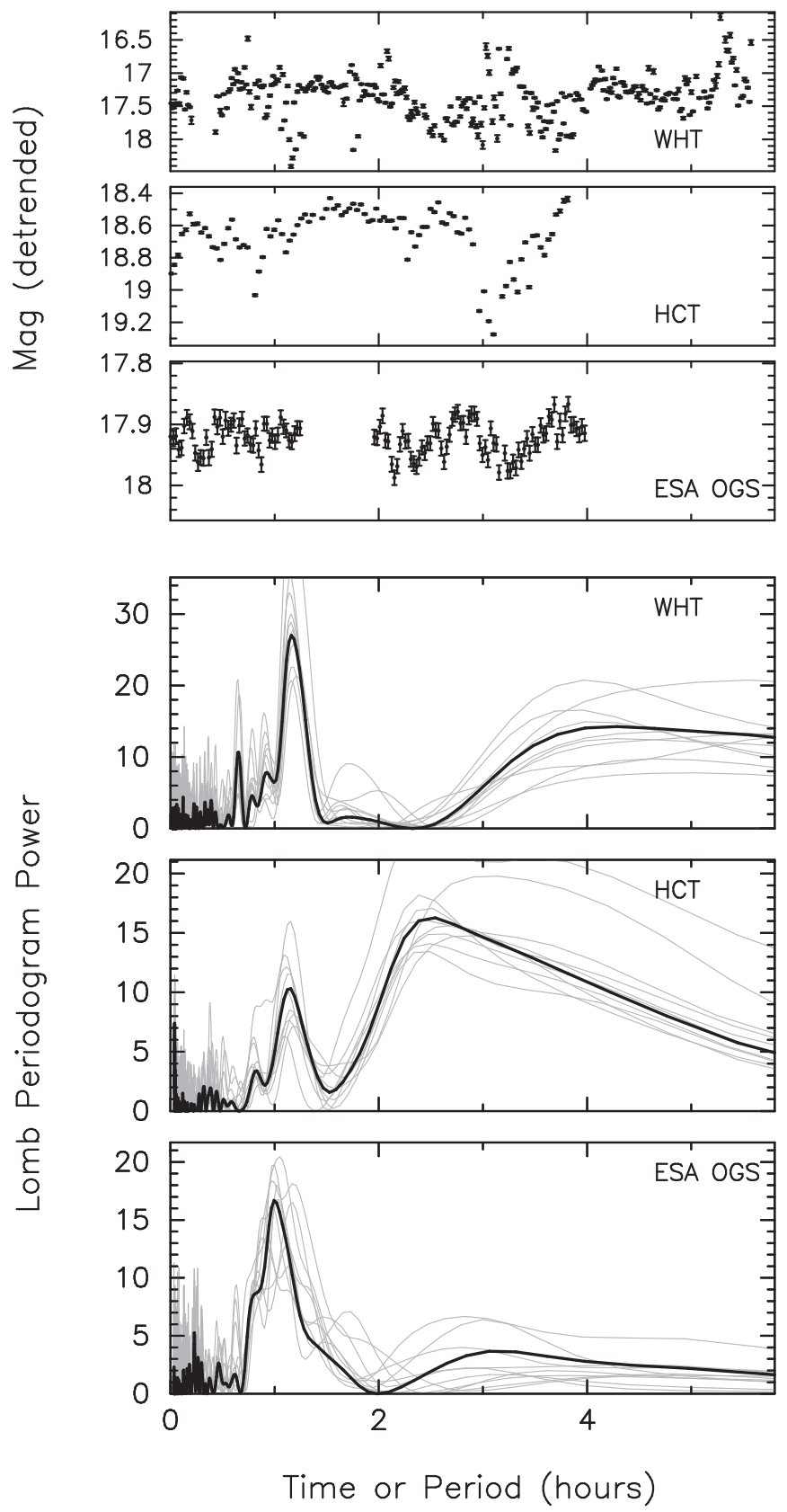

Figure 3. Top three panels: magnitudes vs. time for WHT, HCT, and OGS, after linear detrending. Bottom three panels: the Lomb-Scargle periodogram of spectral power vs. period for these data, with all sets showing a $\sim 1 \mathrm{hr}$ spectral peak, corresponding to a $\sim 2 \mathrm{hr}$ rotation. The gray curves are spectrograms for resamplings of the data, indicating that the spectral signature is robust within each series, despite the noisy nature of the data.

\section{Dynamical Properties}

Gault is a dynamical member of the Phocaea family (Nesvorny 2015), which has been estimated to be (1.2 \pm 0.3$)$ Gyr old (Milani et al. 2017). The Phocaea region is a higheccentricity, high-inclination portion of the inner asteroid belt dominated by S-type asteroids (Carvano et al. 2001) and is bounded by the 3:1 and 4:1 mean-motion resonances (MMRs) with Jupiter and the $\nu_{5}, \nu_{6}$, and $\nu_{16}$ secular resonances.

Using the hierarchical clustering method (Zappala et al. 1990, 1994), we find that Gault also becomes dynamically linked with the overlapping low-albedo Tamara family at a cutoff velocity of $113 \mathrm{~m} \mathrm{~s}^{-1}$, well below the threshold of
$350 \mathrm{~m} \mathrm{~s}^{-1}$ identified for the family (Novaković et al. 2017). Gault's albedo is currently unknown, however, and so its physical association with this family is uncertain.

To assess whether Gault is native to its current location in orbital element space or is a recent interloper like a dynamically evolved Jupiter-family comet, we analyze its long-term dynamical stability. We generate 10 dynamical clones of Gault with Gaussian-distributed orbital elements centered on the object's osculating orbital elements on 2019 February 7, using $\sigma$ values equivalent to the orbital uncertainties $\left(\sigma_{a}=9 \times 10^{-9}\right.$ au, $\sigma_{e}=4 \times 10^{-8}, \sigma_{i}=5 \times 10^{-6}$ degrees). Following the method of Hsieh et al. (2012), we then perform forward integrations for Gault and its clones for $100 \mathrm{Myr}$ (substantially longer than typical dynamical lifetimes for short-period comets; Levison \& Duncan 1994), using the Bulirsch-Stöer integrator in the Mercury $N$-body integrator (Chambers 1999). For broader context, we also perform the same analysis for two sets of 50 clones with $\sigma$ values 10 and 100 times larger than Gault's orbital element uncertainties.

Only one object in the set of clones created using the largest $\sigma$ values is ejected from the solar system (defined as reaching $a>100 \mathrm{au}$ ) during our integrations. All of the other particles in all of the sets of clones remain stable for the $100 \mathrm{Myr}$ integration period, with minimal deviations in the semimajor axis $(\Delta a<0.01 \mathrm{au})$ and osculating elements staying largely within the confines of the Tamara family (Figure 4). These results indicate that Gault is unlikely to be a recently implanted interloper.

\section{Discussion}

The presence of a $\sim 2 \mathrm{hr}$ signature in the three data sets identifies Gault as a superfast rotator near or at the limit of a body with some internal cohesion (Holsapple 2007; Chang et al. 2019). Hence dust emission is strongly suggestive of a rotation-induced event due to the YORP effect, as the object is spun-up by re-radiation forces until the apparent surface gravity is zero, triggering disruption or landslide events (e.g., Scheeres 2015), releasing near-zero-velocity debris that is swept away by radiation pressure. Sudden and brief landslides are in accord with the abrupt dust releases described in Section 4. Because of the large mass of material released, it is likely that these landslides were significant, and that the equatorial velocity of the object is very close to the liberation velocity, i.e., that the surface material is tenuously held to the surface, with a proclivity to rearrange itself. The observed dust velocity of up to $\sim 0.7 \mathrm{~ms}^{-1}$ is in accord with the $\sim 2 \mathrm{~ms}^{-1}$ surface velocity of a $4 \mathrm{~km}$ object rotating with a $2 \mathrm{hr}$ periodi.e., there is no unexplained source of energy. We might see more activations in the future. The low amplitude of the light curve may be explained if the body has already been rotationally reformed to a nearly round, top-like shape, akin to asteroids Ryugu and Bennu.

It is even possible that we are catching Gault in the process of episodic landslide transformation from a Maclaurin spheroid to a slower-rotating Jacobi ellipsoid, perhaps initiated by a collision. In such an event the period would slow down, and the light-curve amplitude should increase. This is consistent with the absence of rotation in the ATLAS data. Further monitoring of the rotation curve before and after any future emission events is warranted. 

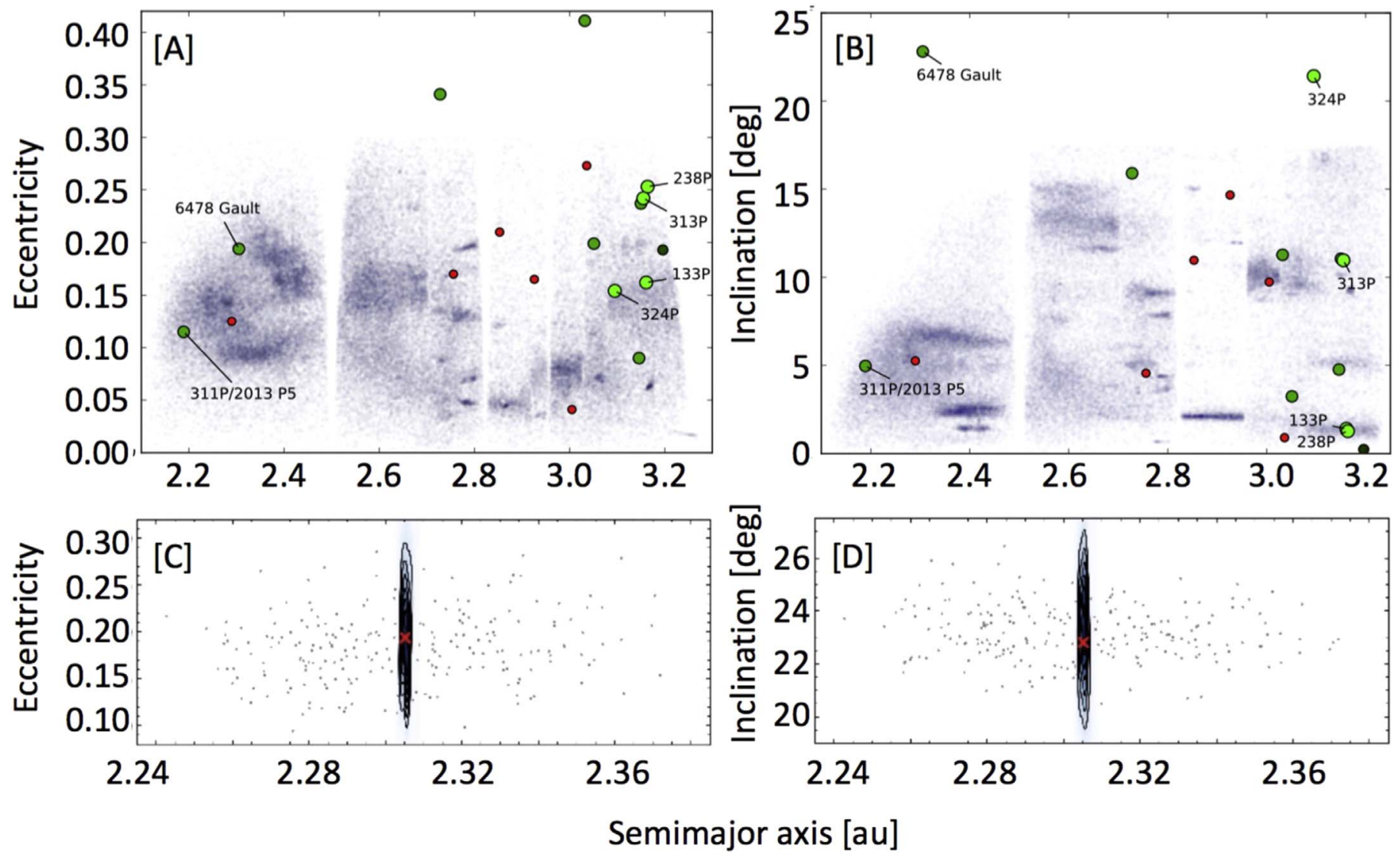

Figure 4. (A, B) Orbital elements. Gault and some other activated asteroids and main belt comets are identified. The red dots correspond to objects likely to have been activated via collision. (C, D) Contour plots (black lines) of intermediate orbital elements in time steps of $10^{4} \mathrm{yr}$ in the osculating semimajor axis vs. osculating eccentricity space and inclination space for 100 Myr forward integrations of Gault and 10 dynamical clones with orbital elements within $1 \sigma$ of Gault's cataloged osculating orbital elements. The original osculating orbital elements of Gault and its clones are marked with a red cross in each panel. The current osculating orbital elements of the members of the Tamara family (small gray dots) are also shown for reference.

The characteristic timescale for a YORP spin-up of a $4 \mathrm{~km}$ object is $\sim 10^{8} \mathrm{yr}$ (Bottke et al. 2006), well within Gault's $\gg 10^{8}$ yr stability time constraint of Section 6.

\section{Summary}

Gault experienced two dust releases occurring around 2018 October 28 and December 30, creating the observed streamers (Jehin et al. 2019; Ye et al. 2019). The October 28 streamer was observed by ATLAS and CFHT. The width of the first streamer is best explained with a maximum emission velocity of $v_{e} \sim 0.7 \mathrm{~m} \mathrm{~s}^{-1}$ in the sky plane. These events were short, with upper duration limits of $\ll 15$ days for the first, and $\ll 5$ days for the second. The size distribution of the dust grains in the streamers follows a power law with an index of $\sim-1.65$. The mass lost in the streamers is $m \approx 7 \times 10^{9}$ and $4 \times 10^{7} \mathrm{~kg}$, respectively.

Dynamical simulations show that Gault is dynamically stable and unlikely to have been recently implanted from elsewhere, ruling out a cometary origin.

The presence of a $\sim 2 \mathrm{hr}$ signature in three data sets identifies Gault as a superfast rotator that likely underwent a YORPinduced rotational disturbance.

We acknowledge the following supporting grants: K.J.M.: NSF award AST1617015, and HST program GO/DD-15678 from NASA through STScI, operated by AURA under NASA contract NAS 5-26555; H.H.H.: NASA Solar System Workings grant 80NSSC17K0723; and A.F.: UK STFC grant ST/ $\mathrm{P} 0003094 / 1$.

This work uses data from the ATLAS project, funded through NASA grants NN12AR55G, 80NSSC18K0284, and 80NSSC18K1575, with the IfA at the University of Hawai' $i$, and with contributions from the Queen's University Belfast, STScI, and the South African Astronomical Observatory.

The INT and WHT are operated on the island of la Palma, by the Isaac Newton Group of Telescopes in the Spanish Observatorio del Roque de los Muchachos of the Instituto de Astrofisica de Canarias.

We thank the staff of IAO, Hanle, and CREST, Hosakote, that made these observations possible. IAO and CREST are operated by the IIAP, Bangalore. We also thank the director and staff of the CFHT, for enabling us to obtain observations on short notice.

Note added. During the proofing stage of this Letter, we learned of an independent paper by Ye et al. (2019b) using ZTF light curve data to reach a similar conclusion that Gault's activity is attributable to rotational disruption or a YORP-driven binary merger.

\section{ORCID iDs}

Jan T. Kleyna (1) https://orcid.org/0000-0002-4734-8878 Olivier R. Hainaut (i) https://orcid.org/0000-0001-6952-9349 Karen J. Meech 느 https://orcid.org/0000-0002-2058-5670 
Henry H. Hsieh (1) https://orcid.org/0000-0001-7225-9271

Alan Fitzsimmons (1) https://orcid.org/0000-0003-0250-9911

Marco Micheli (i) https://orcid.org/0000-0001-7895-8209

Jacqueline V. Keane (1) https://orcid.org/0000-0002-

2021-1863

Larry Denneau (i) https://orcid.org/0000-0002-7034-148X

John Tonry $\mathbb{1}$ https://orcid.org/0000-0003-2858-9657

Aren Heinze (10 https://orcid.org/0000-0003-3313-4921

Bhuwan C. Bhatt (10) https://orcid.org/0000-0003-0174-3829

Devendra K. Sahu (ํ) https://orcid.org/0000-0001-9701-4625

Detlef Koschny (10) https://orcid.org/0000-0001-8690-3507

Ken W. Smith ㄴ) https://orcid.org/0000-0001-9535-3199

Harald Ebeling (i) https://orcid.org/0000-0001-8429-2739

Robert Weryk (i) https://orcid.org/0000-0002-0439-9341

Heather Flewelling (i) https://orcid.org/0000-0002-1050-4056

Richard J. Wainscoat $\odot$ https://orcid.org/0000-0002-

1341-0952

\section{References}

Bottke, W. F., Jr., Vokrouhlický, D., Rubincam, D. P., \& Nesvorný, D. 2006, AREPS, 34, 157

Carvano, J. M., Lazzaro, D., Mothé-Diniz, T., Angeli, C. A., \& Florczak, M. 2001, Icar, 149, 173

Chambers, J. E. 1999, MNRAS, 304, 793

Chang, C.-K., Lin, H.-W., Ip, W.-H., et al. 2019, ApJS, 241, 6

Farnham, T. L. 1996, PhD thesis, Univ. Hawai' $\mathrm{i}$

Finson, M. J., \& Probstein, R. F. 1968, ApJ, 154, 327

Fulle, M., Levasseur-Regourd, A. C., McBride, N., \& Hadamcik, E. 2000, AJ, 119, 1968

Hainaut, O. R., Kleyna, J., Sarid, G., et al. 2012, A\&A, 537, A69

Hainaut, O. R., Boehnhardt, H., Snodgrass, C., et al. 2014, A\&A, 563, A75
Holsapple, K. A. 2007, Icar, 187, 500

Hsieh, H. H., Ishiguro, M., Lacerda, P., \& Jewitt, D. 2011, AJ, 142, 29

Hsieh, H. H., Yang, B., \& Haghighipour, N. 2012, ApJ, 744, 9

Jehin, E., Ferrais, M., Moulane, Y., Pozuelos, F., \& Manfroid, J. 2019, CBET, 4606, 1

Jewitt, D., Hsieh, H., \& Agarwal, J. 2015, Asteroids IV (Tucson, AZ: Univ. Arizona Press), 221

Jewitt, D., Weaver, H., Mutchler, M., et al. 2018, AJ, 155, 231

Kresak, L. 1980, M\&P, 22, 83

Levison, H. F., \& Duncan, M. J. 1994, Icar, 108, 18

Levison, H. F., Terrell, D., Wiegert, P. A., Dones, L., \& Duncan, M. J. 2006, Icar, 182, 161

Lomb, N. R. 1976, Ap\&SS, 39, 447

Maury, A., de Vanssay, J. B., \& Soulier, J. F. 2019, CBET, 4597, 1

Milani, A., Knežević, Z., Spoto, F., et al. 2017, Icar, 288, 240

Moreno, F., Snodgrass, C., Hainaut, O., et al. 2016, A\&A, 587, A155

Moreno, F., Muñoz, O., Gutiérrez, P. J., et al. 2017, MNRAS, 469, S186

Nesvorny, D. 2015, NASA Planetary Data System, EAR-A-VARGBDET-5NESVORNYFAM-V3.0

Novaković, B., Tsirvoulis, G., Granvik, M., \& Todović, A. 2017, AJ, 153, 266

Paunzen, E., \& Vanmunster, T. 2016, AN, 337, 239

Pravec, P., Harris, A. W., \& Michalowski, T. 2002, in Asteroids III, ed. W. F. Bottke, Jr. et al. (Tucson, AZ: Univ. Arizona Press), 113

Scheeres, D. 2015, Icar, 247, 1

Sekanina, Z. 1980, in IAU Symp. 90, Solid Particles in the Solar System, ed. I. Halliday \& B. A. McIntosh (Dordrecht: Reidel), 251

Smith, K. W., \& Denneau, L. 2019, CBET, 4594, 4594

Tonry, J. L., Denneau, L., Heinze, A. N., et al. 2018, PASP, 130, 064505

Vokrouhlický, D., Bottke, W. F., Chesley, S. R., Scheeres, D. J., \& Statler, T. S. 2015, in Asteroids IV, ed. P. Michel, F. E. DeMeo, \& W. F. Bottke (Tucson, AZ: Univ. Arizona Press), 509

Ye, Q., Kelley, M. S. P., Bodewits, D., et al. 2019a, ATel, 12450, 1

Ye, Q., Kelley, M. S. P., Bodewits, D., et al. 2019b, ApJL, 874, L16

Zappala, V., Cellino, A., Farinella, P., \& Knezevic, Z. 1990, AJ, 100, 2030

Zappala, V., Cellino, A., Farinella, P., \& Milani, A. 1994, AJ, 107, 772 